\section{An automated system for maintaining small animals on a water-deprivation regimen}

\author{
PETER C. SAKELLARIS \\ University of Oregon Dental School \\ Portland, Oregon 97201
}

Experiments involving regimented water ingestion often require that watering bottles be manually placed on the Ss' cages at predetermined periods. This procedure often results in cumbersome work schedules for Es and laboratory personnel, especially in studies lasting weeks or months.

The commitment of time and manpower can be reduced through the use of an automatic watering system. Commercial watering systems are available, but are not within the budget limits of many laboratories. However, an inexpensive system which provides the same capabilities as many commercial units can be assembled.

A watering system for ad lib drinking can easily be constructed from standard plastic plumbing pipe and fittings (PVC) or can be purchased as a preassembled unit. In our laboratory, adult rats were housed in individual cages mounted in a Hardco Scientific rat rack (Model RR-2101). The rack contained a factory-assembled PVC system for providing continuous access to drinking water. Asco ${ }^{2}$ three-way solenoid valves were added to regulate watering periods. Figure 1 is a schematic representation of the plumbing system. All solenoid valves were the universal-operation types (Asco 8320A9). Water was provided to the rack system by a flexible industrial hose and connected to Valve 1 by an Aeroquip ${ }^{3}$ coupling. Each valve was connected to the PVC line by a specific orifice. During the period of water deprivation, all three valves were de-energized, i.e., Orifices 1 and 3 were connected and Orifice 2 was closed. Thus, Valves 1 and 3 were open to the atmosphere, and the plumbing system was drained. When the regimen called for access to water, all three valves were energized simultaneously. Valve 1 opened to the water service while Valve 3 closed to the system. Valve 2 opened to the atmosphere, allowing air in the pipes to be forced out by the water filling the system, thus preventing the formation of air pockets. This valve remained open until water, without air bubbles flowed out of Orifice 1 (to the drain), then it was de-energized. A satisfactory operating time for Valve 2, allowing the plumbing system to fill with water and discharge the air from the lines, was found to be $1.5 \mathrm{~min}$. When Valve 2 was closed (de-energized), the system was put

*This work was completed during the author's tenure as a National Research Council Research Associate at NASA, Ames Research Center, Moffett Field, California.

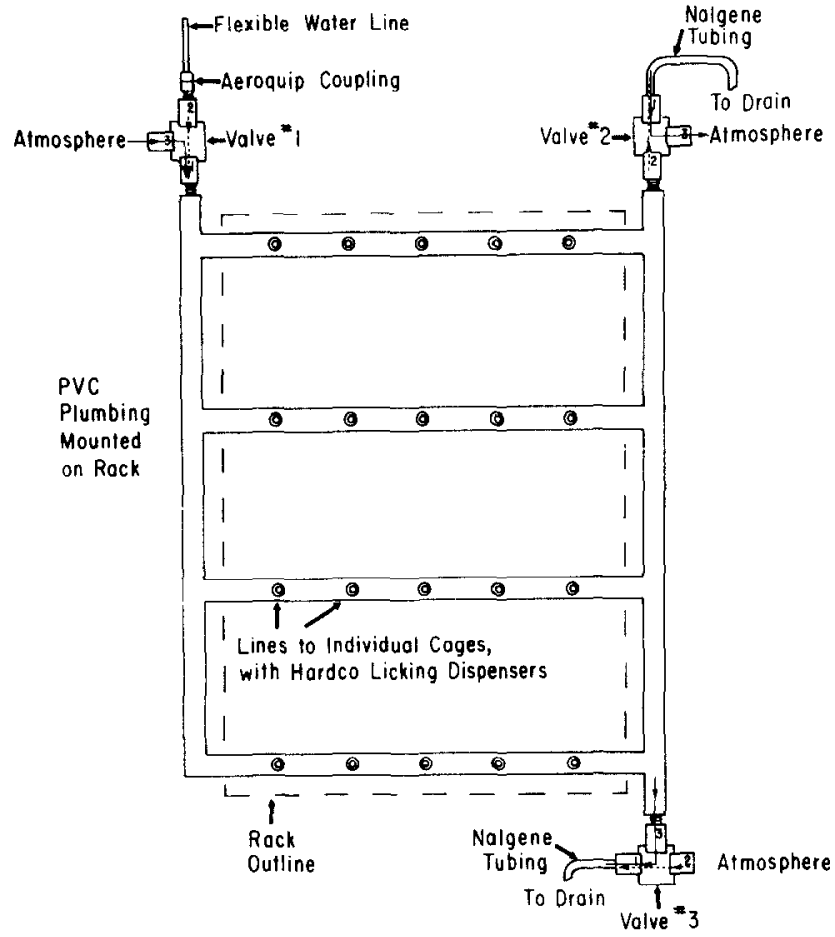

Fig. 1. Schematic representation of the watering system. Solid arrows show valve-flow pattern for de-energized state. Dashed arrows show valve-flow pattern for energized state. Numbers $1,2,3$ in each valve indicate or if ice number.

under line pressure. The other two valves remained energized for the period of time the animals were to be watered. These valves were then de-energized and the system drained rapidly, leaving only a few drops of water in the licking dispensers (see Fig. 1).

The valve activation and sequencing could be accomplished through the use of a small number of behavioral-sciences electronic/electric equipment which is readily available in most psychology laboratories. The author used a 24-h Foringer timer (Model 1181-M11) to activate two other timers of the same type; these in turn activated the valves through accessory relay switches. Electromechanical counters monitored the number of activations of each solenoid, and pilot lights indicated the state of each valve to provide a simple check on the system's operation. Although the valves described herein contained $120-\mathrm{V}$ ac solenoids, they may be purchased with any of five different $\mathrm{dc}$ or four different ac solenoids. ${ }^{2}$

Each valve was enclosed in a large waterproof outdoor recepticle box containing appropriate cutouts for the solenoid leads, water line, or drain lines (refer to Fig. 1). All of the solenoid leads were run through metal conduit to a connector panel at the top of the rack, from which leads were run through flexible conduit to the control equipment. 
The modifications for automating the factory-assembled system, aside from the electronic apparatus, cost less than $\$ 100$. The modifications were completed in one day. This included incorporation of the three solenoid valves, Aeroquip coupling, fittings for connecting the valves to the $\mathrm{PVC}$, recepticle boxes, conduit, and Nalgene tubing. Commercially available systems providing similar or extended capabilities generally add $\$ 500$ to $\$ 600$, or more, to the cost of a preassembled watering unit, excluding the electronic control apparatus. 4

The above-described system has been used with success in two studies of 8 weeks' duration each (Sakellaris \& Vernikos-Danellis, 1974).

\section{REFERENCES}

Sakellaris, P. C., \& Vernikos-Danellis, J. Alteration of pituitary-adrenal dynamics induced by a maintenance regimen. Physiology \& Behavior, 1974, in press.

\section{NOTES}

Ohio.

2. A utomatic Switch Co., Florham Park, New Jersey.

3. A eroquip Corp., Industrial Div., Jackson, Michigan.

4. Hoeltge Incorporated, Cincinnati, Ohio. Environmental Sciences Corp., Edmonds, Washington.
(Received for publication A pril 1, 1974;
revision received May $31,1974$. 